Revista de Psicología de la PUCP. Vol. XIII. No 1. 1995

\title{
APORTES DE GENERO A LA SALUD SEXUAL Y REPRODUCTIVA ${ }^{1}$
}

María Ragúz ${ }^{2}$

This article is about research on the meaning of Gender-Man, Masculinity, Woman, and Femininityin an accidental sample of children, youngsters, adults, and elders from different low SES groups in Lima, Perú. Through content analysis of open interviews it is shown that, for these groups, sex and gender are not being differentiated. Results are interpreted in terms of the implications these conceptualizations of gender have for sexual and reproductive health program interventions, like in family planning, assertiveness training, sex education, women empowerment workshops, male masculinity workshops, and women's assertiveness training and awareness raising programs, as well as interventions in domestic violence and prevention of sexual abuse. The need to trascend gender roles, which sustain gender relations of power and submission in peruvian sociery, is strongly stressed.

Key words: gender, sexual health, reproductive health, masculinity, femininity

Este artículo trata sobre una investigación de significados relativos al Género-¿qué es el Hombre, la Masculinidad, la Mujer, la Femeneidad?- en parte de una muestra accidental de niños, jóvenes, adultos y ancianos de diversos grupos sociales de nivel socioeconómico bajo en Lima, Perú. Mediante análisis de contenido de entrevistas en profundidad se analizan las respuestas, arribándose, entre otras conclusiones, a que en este grupo no se diferencia sexo biológico de género. Se interpretan los resultados en términos de las implicancias que estas conceptualizaciones del género tienen para las campañas de salud sexual y reproductiva como la educación en sexualidad, la planificación familiar, los talleres de "empowerment" ("enpoderamiento") y asertividad para mujeres y de conciencia de masculinidad para varones, o las intervenciones en violencia doméstica y abuso sexual. Se destaca la necesidad de trascender los roles sexuales o genéricos, que sustentan relaciones de inequidad y poder en la sociedad peruana. palabras clave: género, salud sexual, salud reproductiva, masculinidad, femineidad

1 Doctorada en Psicología en la Universidad Católica de Nimega, Holanda y con una Maestría de la Universidad de Wisconsin, Madison, EE.UU. Egresada y catedrática hace dieciocho años de la Pontificia Universidad Católica del Perú donde coordina una Maestría en Psicología y un Taller de Investigación Multidisciplinaria en Género y Salud Sexual y Reproducriva, con el apoyo de la Fundación Ford. Presidenta de la Red Nacional de Educación, Salud Sexual y Desarrollo para Jóvenes (REDESS-jóvenes). Investigadora principal en varios proyectos y consultora.

2 Basado en las conferencias "Masculinidad y femineidad como predictores de logro en mujeres marginales" del Congreso Interamericano de Psicología, Santiago de Chile, julio, 1993; y "Aportes del género a la investigación en salud sexual y reproductiva del adolescente" de la Reunión sobre Salud Integral del Adolescente de FELASSA, México, D.F., marzo de 1994. 

La teoría psicológica del Género constituye una entrada para abordar el tema de la Salud Sexual-Placentera y Reproductiva- (Ragúz, 1993a,b,c,d). Aunque desde los años 70 los estudios han venido demostrando las implicancias de la socialización tradicional de los roles sexuales o genéricos, el aprendizaje social y la construcción de la identidad masculina $y / o$ femenina (Ragúz, 1991a,b,c), no fue hasta la última década en que la Psicología, que ya había venido revisando sus nociones de normalidad y patología, aceptó los hallazgos de la controvertida teoría de la Androginie. Esta teoría psicológica, vinculada a las conceptualizaciones feministas de la Teoría del Género, sostiene que la masculinidad y la femineidad no son excluyentes de uno u otro sexo, sino que son dimensiones que coexisten, en distinto grado, al interior de cada persona, hombre o mujer, y que estos esquemas genéricos van a determinar el comportamiento.

Así, los manuales de Psicología contemporánea plantean que la socialización tradicional hacia el hombre masculino y la mujer femenina ha dejado de ser el ideal de las culturas modernas, que los roles deben superarse y que debe redefinirse el ideal de ser humano. Pero en todos los países $-\mathrm{y}$, más aún, en nuestros países latinoamericanos signados por el machismo de la Conquista- la tradicional socialización diferencial de los sexos y la construcción del género siguen siendo una realidad.

Los avances de la Teoría de Relaciones del Género no han tenido, aún, impacto en la teoría psicológica. Aunque los roles tradicionales son cuestionados, no se aprecia una claridad en la conceprualización de su relación con situaciones de poder y discriminación de esferas reales de influencia social.

¿Por qué es que nos preocupa que los niños sigan formándose en lo que la cultura define como masculino, y las niñas, en lo femenino? Porque en base a los estereotipos del rol sexual se construye la identidad de las 
personas, una identidad fuertemente marcada por el sexo y el género. $Y$ esa identidad psicosocial es el resultado y, a la vez, la base, de una estructura socioeconómica, política, cultural, de relaciones de género, de relaciones de poder y de discriminación. ¿Cómo así?

Las investigaciones con diversas poblaciones del mundo (Ragúz, 1993b, 1991 a,b) muestran que la masculinidad psicológica de una persona, varón o mujer, predice de manera significativa no sólo rendimiento matemático, sino habilidad matemática y ansiedad hacia las matemáticas. Predice también rendimiento en tareas espaciales. Asi mismo, dependiendo de si la masculinidad es alta, también lo será la motivación de logro y la expectativa de éxito; mientras que si la masculinidad es baja, habrá temor al fracaso, temor al éxito, conflicto de logro, locus de control externo, incapacidad o desesperanza aprendida.

La masculinidad es un predictor exitoso de asertividad y confianza en si mismo/a. Las personas altamente masculinas son activas, toman iniciativa, corren riesgos calculados, defienden sus posiciones, toman decisiones y tienen la capacidad o la conducta de liderazgo. Lo inverso es cierto cuando la masculinidad es baja, predominando la pasividad, la sumisión, la dependencia, el miedo, la influenciabilidad, la poca competitividad (Ragúz, 1991c,d).

Las personas poco masculinas tienen menores metas educativas, mayor estereotipia en la elección de las vocaciones y ocupaciones, tradicionalismo de intereses, ausentismo escolar.

La masculinidad se asocia, igualmente, con variables de ajuste y salud mental. En especial, la baja masculinidad en hombres y la baja masculinidad combinada con baja femineidad en ambos sexos, se asocian con un ajuste psicológico pobre. La masculinidad, respecto de variables negativas, se asocia únicamente con estrés. Por su parte, la femineidad predice falta de asertividad, neuroticismo, baja autoestima, inseguridad, auto-desprecio, incapacidad aprendida, depresión. Un estudio realizado con psicoterapeutas de orientación dinámica y estudiantes de psicología en el país, mostró que el perfil de salud mental ("persona sana, bien adaptada") coincide casi perfectamente con el ideal de Hombre y no tiene casi coincidencia con el ideal de Mujer (Ramos, 1987). 
¿Por qué se da esto? El análisis matemático de la estructura latente de los conceptos de masculinidad y femineidad (Ragúz, 1991a), muestra que mientras que femineidad es un constructo unidimensional que gira en torno a maternidad y a maternalidad o cuidado, la masculinidad tiene, por lo menos, dos dimensiones: masculinidad interna (autoconcepto y autoestima altos, independencia, asertividad, seguridad en sí mismo, etc.), y masculinidad externa (dominio, agresión, conducta de liderazgo sin tener habilidades de líder). Depende de cómo estas dimensiones se combinen al interior de cada persona, cómo ésta se comporte, ya que marcan su identidad, son parte crucial de su autoconcepto y autoestima.

Tal como se conciben la masculinidad y la femineidad en el mundo actual, es la masculinidad interna lo que usualmente predice éxito, y, en contadas ocasiones, también la masculinidad externa (Ragúz, 1993a). Esto puede explicarse por cómo se define y mide masculinidad, dándose una tautología al definirla como buen autoconcepto y alta autoestima. El autoconcepto y la autoestima social, así como la masculinidad, suelen ser mayores en los varones. Y la teoría psicológica ha probado que el autoconcepto mediatiza en rendimiento. Las expectativas de éxito son mayores y el logro y rendimiento, mejores, cuando combina con un locus de control interno, que suele ser más característico de los varones. Por ejemplo, un estudio con adolescentes mujeres urbano-marginales de Lima mostró que a menor autoestima, más tendencia a tener locus de control externo y mayor conformismo (Lira, 1991).

Una posible explicación de esta conceptualización y diferencial valoración de la masculinidad y la femineidad la dan las Teorías de las Relaciones de Género y de los Roles Sexuales. La identidad -y, en particular, su componente de identidad sexual biológica y genérica- se construyen en el desarrollo humano a lo largo de la vida. El ser humano es socializado al interior de un sistema social con determinados valores e ideología. En las sociedades, mayoritariamente tradicionales en su concepción del género, la identidad sexual, en sentido amplio, parte de definiciones de sexualidad y de salud que responden a una visión básicamente biológica y médica, donde se enfatiza la sexualidad reproductiva (en especial, la heterosexualidad monogámica al interior del matrimonio y para la preservación de la familia). A estos roles reproductivos complementarios corres- 
ponden roles de género supuestamente complementarios y dicotómicos, excluyentes, donde la masculinidad es un atributo de los hombres y la femineidad, de las mujeres.

Los roles e identidades genéricos son, en realidad, el pilar sobre el cual descansan las relaciones de poder y sumisión, el tratamiento diferencial de las personas en base a su sexo biológico y a supuestas diferencias innatas, y la discriminación de la mujer (y de otros grupos) de esferas de poder real. Una de estas discriminaciones es la educación formal, tal como lo muestra el reporte de Population Action International de 1993, comparando 116 países (95 por ciento de la población mundial; Perú fue el único país Sud Americano no incluído por falta de estadísticas adecuadas). El reporte estima que en 1990 la brecha educacional entre sexos era de 76 millones de mujeres jóvenes, siendo más marcada en Asia del Sur, Oriente Medio y Africa. Para cerrar la brecha educacional en el año 2005, se calcula un costo de 18 billones de dólares.

¿Cómo se conceptualizan la femineidad y la masculinidad en el Perú? Aunque un estudio transcultural de hace más de una década (Williams y Best, 1982) catalogó al Perú como "medianamente estereotipado", es cuestionable la generalizabilidad de los resultados por el sesgo que tuvo el muestreo. La estereotipia del género parece ser aún predominantemente tradicional, por lo que hemos podido encontrar en una revisión de estudios sociológicos, antropológicos y psicológicos realizados en el país (Ragúz y Pinzás, 1993). Lo mismo encontramos en una serie de investigaciones con universitarios y preuniversitarios limeños que hiciéramos a lo largo de casi una década (Ragúz, 1991a). El Taller Andino que, con el auspicio de Pathfinder International y la Universidad de Lima, realizáramos con decenas de profesionales de la salud y educación que trabajan a nivel nacional con jóvenes, adolescentes y educadores en sexualidad, arribó a la misma conclusión (Fernández, Ragúz y Sebastiani, 1993).

Una investigación que estamos realizando con el apoyo de la Fundación Ford (Ragúz, en preparación), parte de cuyos resultados descriptivos fueron presentados en el Congreso Interamericano de Psicología, Santiago de Chile, en Julio de 1993 (Ragúz, 1993c) y en la Reunión sobre Salud Integral del Adolescente de FELASSA en México,D.F., en Marzo de 1994, permite ilustrar el tradicionalismo en la definición de los masculino y lo 
femenino que persiste en diversos sectores de la sociedad peruana. Aunque se trata de muestras no sistemáticas, el estudio tiene alta validez interna y permite visualizar tendencias. Además, es un ejemplo de confluencia de métodos (Katzko y Ragúz, 1994), recolectando información cualitativa y sistematizándola de modo que se posibilita el análisis cuantitativo y la respuesta a las preguntas exploratorias: ¿qué es Masculinidad y Femineidad para diversos segmentos de la población peruana? ¿Son o no lo mismo que Hombre y Mujer, respectivamente? De ahí que nos parezca interesante presentar acá el resultado de un primer análisis de los datos, con una parte de la muestra, los primeros casos recogidos y ya sistematizados.

\section{Método}

Se trata de un estudio descriptivo y exploratorio, que reporta parte de los resultados -62 casos- obtenidos con muestras accidentales de más de 600 personas de Lima y provincias, de diversas características sociodemográficas.

Aquí se expondrán los resultados descriptivos de estos primeros 62 casos analizados. Se trata de peruanos de edad variada ( 9 á 75 años), religión predominantemente Católica, de diversos tipos y niveles de educación mixta o segregada, de diverso estado civil y tipo de familia, de diversa procedencia y tiempo de residencia en Lima, con ocupaciones variadas (desde empleadas del hogar, sus hijos, cadetes en Servicio Militar, promotores comunales, jubilados, escolares, pre universitarios y universitarios y amas de casa (ver Cuadro 1). Predomina en los casos aquí presentados el nivel socioeconómico bajo y muy bajo (pobreza), al menos por los indicadores de ocupación, lugar de residencia, tipo de escolaridad, e historia migratoria.

Se empleó la técnica cualitativa de la entrevista individual en profundidad, indagando por el significado de lo masculino y lo femenino empleando la "técnica del marciano", diciendo: "Imagínate que viene un marciano a la Tierra. No sabe nada de este mundo y tú quieres explicarles que hay hombres y mujeres y cómo son, en qué son diferentes y en qué se parecen. ¿Qué le dirías?" Primero, "¿Qué es un hombre? "Luego que el entrevistado terminaba esta respuesta se le preguntaba “Qué es una mujer?" Seguidamente, “Qué es la masculinidad, lo masculino?" Luego, “' Y la femineidad, 
lo femenino?" Era posible repreguntar para profundizar en las respuestas, pero evitando sesgarlas, hasta tener una idea clara de los conceptos.

Los entrevistadores fueron estudiantes de Psicología que registraron literalmente las respuestas. Para el análisis de resultados, la autora codificó las entrevistas en base al análisis de contenido de más de mil respuestas que se sistematizaron en 33 categorías de Femineidad (tradicionalmente definida), 38 de Masculinidad y 3 de Conciencia de Relaciones de Género. Esta categorización permite la cuantificación y el análisis estadístico, aunque acá nos limitaremos a estadística descriptiva.

Este es un reporte parcial de los resultados ya que el análisis multivariado, tomando en cuenta las diversas variables sociodemográficas, ha de realizarse próximamente con la totalidad de la muestra, bajo el auspicio de la Fundación Ford.

Cuadro 1: Composición de la muestra

\begin{tabular}{|llrrr|}
\hline \multicolumn{1}{|c}{$\begin{array}{c}\text { ocupación/ } \\
\text { descripción }\end{array}$} & edad & \multicolumn{2}{c|}{ frecuencia } \\
mujeres & varones & total \\
\hline promotores comunales & adulta & 14 & 4 & 18 \\
ex-dirigentes comunales & adultos & 2 & 2 & 4 \\
“avioneritos" FFAA Arequipa & jóvenes & 0 & 5 & 5 \\
empleadas del hogar, Arequipa & jóvenes & 5 & 0 & 5 \\
universitarios UNMS & jóvenes & 6 & 3 & 9 \\
hijos de ex-dirigentes comun. & niños 9/10a & 3 & 2 & 5 \\
hijos empl. hogar Arequipa & niños 9/10a & 5 & 5 & 10 \\
\multicolumn{1}{|c|}{} & & 37 & 25 & 62 \\
\hline
\end{tabular}

\section{Resultados}

Lo primero que encontramos es que la mayoría de los entrevistados y entrevistadas, al ser preguntados "Qué es la Masculinidad?", respondían "Hombre"; y "Qué es la Femineidad?", "Mujer". Es decir, no diferenciaban entre los conceptos de Mujer y Femineidad o de Hombre y Masculinidad, no siendo posible tratar los datos por separado. 
Encontramos más definiciones y atributos de Masculinidad que de Femineidad y muy pocas respuestas que espontáneamente hicieran alusión a una Conciencia de Relaciones de Género (en términos de que las diferencias no físicas entre los sexos son diferencias culturales, aprendidas, que hay machismo y discriminación, que debería haber igualdad de derechos y deberes). Por el contrario, hubo respuestas que apuntaban no sólo a la creencia en diferencias innatas e inmutables, sino a un explícito acuerdo con el "status quo" y hasta con la dominación del hombre.

Lo Femenino (más en términos de Mujer que de Femineidad) fue definido muy poco en base a aspectos sexuales físicos primarios y secundarios, como los órganos sexuales y reproductores, mamas o busto y la voz (ver Tabla 1). Más bien predominaron alusiones a características comportamentales como inseguridad, temor, estar indefenso/a, debilidad, poca fuerza, pasividad y sumisión, cuidado de los hijos, maternidad y maternalidad, rol doméstico y de esposa.

Tabla 1: Definidores de lo femenino (mujer/femineidad) [en porcentajes]

\begin{tabular}{|lr|}
\hline Definidores & $\%$ \\
\hline ASPECTOS FISICOS PRIMARIOS Y SECUNDARIOS & 18 \\
\hline -mamas, voz, órganos sexuales & \\
\hline CARACTERISTICAS COMPORTAMENTALES & 82 \\
\hline -inseguridad, temor, estar indefenso/a, debilidad, poca fuerza, & \\
pasividad, sumisión & 15 \\
-cuidado de los hijos, maternidad y maternalidad & 8 \\
-rol doméstico y de esposa & 4 \\
-delicadeza (modales,buena conducta pública, elegancia y distin- & \\
ción, suavidad) y delicadeza como fragilidad y falta de fuerza & 7 \\
-afectividad (afectuoso, cariñoso, expresar sentimientos) & 5 \\
-diferencias intelectuales negativas para la mujer & \\
(menor inteligencia, o atención) & 5 \\
-diferencias intelectuales positivas para la mujer & 5 \\
(más inteligencia, viveza, creatividad) & 2 \\
-mayor moral (conflabilidad, sinceridad, honestidad, moralismo) & 2 \\
-mayor responsabilidad, lealtad y consecuencia & 6 \\
-discriminación, inequidad & 23 \\
-otros (variados) & \\
\hline
\end{tabular}


Lo Femenino se asociaba, también, con delicadeza. Delicadeza tanto en términos de modales, como en términos de fragilidad y falta de fuerzas. $\mathrm{Y}$ lo Femenino se asociaba, además, con afectividad en términos de afectuosidad, cariño, expresividad de sentimientos.

Para quienes asociaban lo Femenino con diferencias intelectuales o cognitivas, la mitad atribuía menor inteligencia o atención a las mujeres; la otra mitad, más inteligencia, viveza y creatividad.

Un pequeńo porcentaje de respuestas aludía a mayor moral (confiabilidad, sinceridad, honestidad, moralismo) en las mujeres, y a su mayor responsabilidad, lealtad y consecuencia.

Contados casos asociaron Femineidad con discriminación e inequidad, e, inclusive, algunas respuestas eran en la línea de que no creían posible un cambio o que estaban de acuerdo con la situación actual.

Tabla 2: Definidores de lo masculino (hombre/masculinidad) [en porcentajes]

\begin{tabular}{|lr|}
\hline Definidores & $\%$ \\
\hline ASPECTOS SEXUALES FISICOS PRIMARIOS Y SECUNDARIOS & 11 \\
-voz gruesa, órganos sexuales y reproductores & \\
\hline CARACTERISTICAS COMPORTAMENTALES & 89 \\
\hline -rudeza y agresividad (tosquedad, ser poco delicado, recio y rudo; & \\
violencia, abuso y agresividad juegos de guerra y peleas) & 6 \\
-mayor fuerza física & 6 \\
-forma de vestir y corte de pelo & 7 \\
-mayor sueldo & 5 \\
-rol instrumental & 3 \\
-rol de pareja o esposo & 2 \\
-paternidad y la paternalidad & 1 \\
-afectividad como control y represión de emociones y sentimientos & \\
(no llorar, menor expresividad, menor sentimentalismo, menor afecti- & \\
vidad, más dureza, insensibilidad, ecuanimidad) & 5 \\
-discriminación a favor del hombre (más preparados, libres, poder, & \\
dominio, mandan y dan órdenes) & 6 \\
-otras (variadas) & 48 \\
\hline
\end{tabular}


En cuanto a lo Masculino -más en términos de Hombre que de Masculinidad-, primaron también respuestas en torno a aspectos comportamentales. Los aspectos sexuales físicos primarios y secundarios giraron en torno a voz gruesa, y menos, en torno a órganos sexuales y reproductores y fuerza física (ver Tabla 2).

Los aspectos comportamentales masculinos más mencionados fueron: rudeza y agresividad, tosquedad, ser poco delicado, recio, rudo (una cuarta parte de las respuestas haciendo alusiones a violencia, abuso y agresividad, y a juegos de guerra y peleas). También se relevó la estereotipia en la ropa $y$ el corte de pelo.

El mayor sueldo de los hombres fue reportado por algunos, lo mismo que el rol instrumental, de proveedor. El rol de pareja o esposo apenas se mencionó. La paternidad y la paternalidad no llegaron ni a un uno por ciento de las respuestas.

En cuanto a la afectividad, se definió lo masculino como control y represión de emociones y sentimientos, no llorar, menor expresividad, menor sentimentalismo, menor afectividad, más dureza, insensibilidad y ecuanimidad.

Muy pocos asociaron Masculinidad con discriminación, siendo entendida como mayor preparación para todo, mayor libertad y acceso a lo público y a posiciones de poder y dominio en la sociedad, así como ser mandones y dar órdenes.

\section{Discusión}

En base a los resultados encontrados vemos algunas tendencias interesantes. Lo primero es que Femineidad y Masculinidad -Género psicológico- no se distinguen claramente de Sexo Biológico, Mujer y Hombre, respectivamente.

Segundo, se definen, básicamente, por atributos de personalidad y conducta, más que por rasgos físicos. Pero un definidor esencial de lo Masculino y de los Hombres es la fuerza muy estrechamente asociada a la idea de daño. Redondean el concepto de Masculinidad, el rol instrumental de proveedor económico y mediador de lo privado con lo público, así como 
el control de las emociones. Se vé también la atribución casi nula del rol paternal.

Femineidad y Mujer son definidas en base al rol maternal y maternante y doméstico, pero se enfatiza más el aspecto de vulnerabilidad.

Tercero, a pesar de que son diferencias comportamentales lo que predomina en la percepción de la Masculinidad y la Femineidad, hay una creencia en diferencias innatas e inmutables que justifican los roles y muy pobre conciencia de discriminación, de las relaciones de Género y de la construcción de la identidad genérica.

Cuarto, podemos concluír que, en los grupos estudiados, prevalece el tradicionalismo en la estereotipia del género en la conceptualización de Femineidad y Masculinidad, y de Mujer y Hombre. Al parecer, el tradicionalismo no es privativo de los universitarios y preuniversitarios o de mujeres de sectores medios limeńos o de educadores de adultos a nivel nacional, o de ciertos centros escolares de Lima, diurnos y nocturnos, sectores estudiados hasta la fecha, sino que parecen caracterizar a diversos sectores de la población peruana, aunque requerimos de muestras más representativas para poder afirmarlo.

El análisis multivariado con los datos de toda la muestra permitirá darle mayor validez externa (generalizabilidad) a estos primeros resultados, detectar diferencias debidas a la interacción de variables sociodemográficas, establecer patrones y tendencias que permitan mayores generalizaciones y el esbozamiento de algunas hipótesis de explicación.

Habíamos hablado antes de que la Masculinidad predice éxito en una serie de áreas personales y sociales, mientras que la Femineidad aporta poco o negativamente. En cuanto a educación (motivación, intereses, metas, expectativas), vimos que la Masculinidad y la Femineidad signan a las personas en sus expectativas y comportamientos.

Un ejemplo lo encontramos en el reporte de Population Action International -antes mencionado- el cual sostiene que los padres tienen menores expectativas educacionales para las hijas, a quienes se les asocia con un matrimonio más temprano y menores oportunidades laborales, y quienes se hacen cargo de lo doméstico por lo que se las mantiene en el 
hogar. El embarazo temprano (altamente predecible en medios tradicionales) termina siendo otro motivo más para la brecha educacional. Las tasas de fertilidad y de mortalidad infantil van de la mano con el analfabetismo y nivel educativo y la pobre calidad de muchos de los servicios de planificación familiar y de salud materno-infantil y de la mujer existentes en los países.

Tomando en cuenta las diferencias entre hombres y mujeres en su matrícula en Primaria y en Secundaria, y el número promedio de años de escolaridad, Population Action International construyó un Indice de Escolaridad de la Mujer que permitió comparar la casi totalidad de países, viéndose que mientras que Francia y Canadá tienen más de 99 sobre 100, y Uruguay, Chile, Colombia, Panamá, Jamaica, Ecuador, Venezuela, Nicaragua, República Dominicana, México, Costa Rica tienen un índice entre 89 y 75 sobre 100 . Brasil, El Salvador, Bolivia, Haití tienen entre 74 y 60. Los países de Oriente Medio y Africa son los de menor índice, siendo los más bajos Chad, Guinea, Yemen, Afganistán y Mali, con índices entre 21 y 24 sobre 100. Hay un cuarto de países (más de 25) que tienen un promedio de escolaridad femenina que no llega ni a un ańo de escolaridad. En casi un diez por ciento de los países no se llega ni a 60 mujeres por cada cien hombres que estudian Primaria, y casi en un veinte por ciento de países las mujeres en Secundaria no llegan a ser ni el 60 por ciento de los hombres registrados.

Una de las conclusiones de estudios como los de Population Action International es que hay que potenciar -"empowerment" o "enpoderamiento"- a las mujeres, en especial, a través de su educación. Y, podemos agregar, es necesario hacerlo incorporando los aportes de la Teoría de los Roles Sexuales y las Relaciones de Género.

Desde las concepciones de género tradicionales que aún prevalecen en sociedades como la nuestra, no sorprenden los hallazgos transculturales de que las personas femeninas -principalmente mujeres, por probabilidadesson las más tradicionales en sus actitudes hacia la sexualidad, las que menos tienden a una protección anticonceptiva efectiva, las que más tienen embarazos indeseados, las que menos abortan hijos indeseados, las que más conductas sexuales de riesgo tienen, las que más experimentan abuso sexual $\mathrm{y}$ otras formas de violencia. Son las mujeres femeninas las que más hijos 
tienen y quieren tener, las que presentan menores metas y expectativas educacionales y menores logros en esta área, las que menos ganan y más satisfechas están con su estatus bajo y con su vida, y menos conciencia tienen de la discriminación y la marginación del poder, o menos creen en la posibilidad de un cambio (Ragúz, 1991d).

Es así que los hallazgos y desarrollos en las Teorías del Género y de los Roles Sexuales deben tenerse en cuenta para el diseño de las intervenciones en salud y educación sexual (Ragúz y Bendezú, 1992). Como seńala Gabriela Rodriguez (1993), en su resumen del Simposio Latinoamericano de Planificación Familar de México 1992, "Experiencias de Género en los Programas de Planificación Familiar en América Latina", la mayoría de programas de planificación familiar han sido diseñados sólo para la mujer. Los programas de salud y educación centrados en el binomio madre-niño dejaron de lado al hombre en los servicios de planificación familiar y a la mujer, salvo la mujer-madre o embarazada, atendiendo a su salud sexual solamente reproductiva.

Son las mujeres femeninas las que menor autoestima tienen, lo que va de la mano con baja asertividad. De ahí que las estrategias de "enpoderamiento" sean indispensables. Pero no se trata de reforzar el yo psicológico, la autoestima y el autoconcepto con frases positivas y ejercicios de valoración. Se requiere un cambio estructural en las relaciones y una herramienta indispensable es habilitar a la persona para su independencia económica, que es clave para la independencia emocional. Como se vé en los estudios sobre violencia doméstica (que desmienten mitos como que la mujer disfruta del castigo o ama a la pareja y por eso no rompe el círculo de abuso), cuando a la mujer se le permite subsistir sin depender de la pareja victimizadora, ella inmediatamente lo abandona (Ragúz, 1992a, capítulo "Agresiones Sexuales").

Es de esperarse que los hombres masculinos, y, en particular, los de alta masculinidad externa con poca masculinidad interna y poca femineidad autopercibida, sean los más machistas, abusadores e irresponsables. Y cabe esperar que este tipo de hombre sea una suerte de ideal cultural en las sociedades tradicionales. ¿Cómo trabajar con estos hombres en las intervenciones? No son, precisamente, los que van a acudir a un programa de manera espontánea. 
Sabemos que son los sectores menos favorecidos de la sociedad peruana, $y$, en especial, las mujeres, los que más sufren de una socialización tradicional de los roles sexuales y los que en más desventajosa posición se encuentran respecto al cambio significativo de sus condiciones de vida y de identidad psicológica, no sólo por dificultades de oportunidades externas y medios materiales que explican actitudes de incapacidad aprendida y desesperanza, sino también por el tradicionalismo pervasivo que sólo permite cambios superficiales en las relaciones entre los géneros.

Las mujeres, y en especial, las de menores estratos socioeconómicos, están más limitadas en sus oportunidades de desarrollo en la vida desde este tradicionalismo. Los esfuerzos por mejorar sus condiciones de vida deben apuntar también a un cambio profundo en la identidad sexual de género.

Otro problema relativo a las Relaciones de Género y la salud sexual y reproductiva es el de la violencia contra la mujer en la relación vertical con muchas de las "autoridades" médicas y del personal de salud. La mujer es considerada una paciente en el mal sentido del término (igualando salud con ausencia de enfermedad y no con calidad de vida), como una usuaria -objeto y no sujeto de servicios- con la que se establece una relación de poder (por ejemplo, en muchas de las consultas de Planificación Familiar, en las Maternidades, en los consultorios clínicos).

Por orra parte, el enfoque de género en la intervención educativa en sexualidad no significa hablar sólo de roles tradicionales y de que el varón puede, eventualmente, realizar tareas domésticas "femeninas" o que la mujer "puede" estudiar y trabajar.. Género supone relaciones de poder y una construcción psicológica de roles encuadrados en esas relaciones, que las sustentan y eternizan. Cambios insustanciales no llevan a relaciones de equidad.

Un enfoque de Género permite entender, mejor la dificultad del cambio actitudinal y comportamental en los adolescentes que llevan programas tradicionales de educación en sexualidad, a pesar de que sus conocimientos y nivel de información sobre sexualidad hayan mejorado notablemente. No es sólo un problema de información. Y una de las variables que creo contribuye sustancialmente a dificultar el cambio es la 
visión relativamente tradicional de los roles, donde maternidad y maternalidad son los definidores por excelencia de lo femenino, aunados a sumisión, dependencia, pobre autoestima, locus de control externo y demás, que imposibilitan un cambio en la relación de poder con el mundo masculino (que, en sociedades como la peruana, está teñido, además, del machismo signado por el abuso, la violencia y el descompromiso con la prole).

La coresponsabilidad es utópica cuando sólo la adolescente, y no también el varón, es vista como en riesgo de embarazo. La sexualidad responsable es una palabra hueca cuando las púberes son iniciadas por seducción o violación de familiares o figuras cercanas, aunque el embarazo pueda ocurrir luego de ańos de una historia de abuso sexual.

La protección anticonceptiva se dificulta cuando la cultura, muchas veces fuertemente influenciada por las religiones, sigue sosteniendo que el rol de la mujer es, en esencia, la maternidad, y que en los jóvenes la abstinencia permanente es lo razonable, o que los métodos naturales son efectivos (cuando éstos suponen una relación democrática o un poder de negociación que no existe en la mayoría; de parejas). Igualmente, ¿qué sucede con los jóvenes cuya orientación sexual no es la de la mayoría, cuando a pesar de que la psicología moderna haya dejado de hablar de desviaciones, perversiones o anormalidades en sentido clínico, la cultura sigue reprimiendo su expresión y discriminando personas sobre la preconcepción de que la sexualidad es, en esencia, reproductiva? Desde esa sexualidad reproductiva hablamos de planificación "familiar", de "normalidad" y adaptación, de salud sexual reproductiva, dejando de lado la salud sexual placentera no reproductiva.

¿Cómo planificar campañas para adolescentes sin visión de futuro, con pensamiento omnipotente ("a mí no me va a pasar"), de fuerte impulsividad, de relaciones eventuales difíciles de planificar, quienes pueden asociar sexo con placer pero rara vez tienen presente, en su real dimensión, la posibilidad del embarazo indeseado?

¿Cómo desarrollar campañas que no tomen en cuenta, por ejemplo, las relaciones de poder entre los géneros? Los más recientes trabajos en prevención del SIDA -un problema especialmente sensitivo para las po- 
blaciones adolescentes- señalan las dificultades implícitas para la prevención cuando las relaciones de género se caracterizan por inequidades y relaciones de poder/sumisión en su infinidad de expresiones.

Por ejemplo, en "La triple amenaza: Mujeres y SIDA" de Abia y SOS Corpo (1993) se toca el problema de mayor riesgo de contagio en la mujer y se cuestiona su poder de negociación para el sexo seguro, en especial, de la mujer pobre. Al hablar de prevención y estatus de la mujer, se puntualiza que decir "no" al sexo no deseado, al sexo sin protección o a la maternidad indeseada es también decir "no" a la desigualdad, la discriminación y a la falta de opciones de la mujer, ya que es claro que a mayor superioridad social y económica, menor situación y comportamiento de riesgo.

Es necesario que se deje de hablar de Género como equivalente de "sexo socializado", de una relación unívoca masculinidad=hombre, femineidad=mujer que es falsa y que entrampa la acción de cambio. Es necesario que un enfoque de Género se incopore a las acciones de intervención en salud sexual y reproductiva con los adolescentes. Aunque el enfoque de Género no garantice el éxito, el no incorporarlo prácticamente garantiza el fracaso, cuando lo que se busca es un cambio real y durarero.

\section{Referencias}

Fernández, M., Ragúz, M., y Sebastiani, A. (1993). Memoria del Taller sobre Salud Sexual y Reproductiva en Jóvenes y Adolescentes. Lima: Universidad de Lima/Pathfinder Int. Documento interno.

Katzko, M. y Ragúz, M. (1994). Guía metodológica para la investigación psicológica en el Tercer Mundo. Nimega, Holanda: KUN; Lima: PUCP. En preparación.

Lira, L. (1991). Variables demográficas, autoestima, locus de control y conformismo en adolescentes en relación a sus actitudes hacia la mujer. Tesis de Bachillerato en Psicología, PUC, Lima.

Panos/Abia/sOS Corpo (1993). A triple amenaca: Mubljeres \& AIDS. Brasil. Population Action International (1993). Closing the gender gap: Educating girls. 1993 Report on Progress towards world population stabilization. WA, D.C. 
Ragúz, M. (en preparación). Construcción social y psicológica del género en diversos estratos poblacionales peruanos. Investigación en proceso, Lima: PUCP/Fundación Ford.

Ragúz, M. (1994). Aportes del Género a la Investigación en Salud Sexual y Reproductiva del Adolescente, Mesa Redonda de Investigación en la Reunión Internacional de FELASSA, México D.F., Marzo. CENDOC Psicología PUC, Lima.

Ragúz, M. (1993a). Masculinidad y femineidad como predictores de logro en mujeres marginales, Mesa Redonda "Psicología y Pobreza", Congreso Interamericano de Psicología, Santiago de Chile, Julio 1993.

Ragúz, M. (1993b). Hacia una reconceptualización del género. Material de apoyo, CENDOC Psicología PUC, Lima.

Ragúz, M. (1993c). Celeste, rosa o amarillo? Revista Crianza, Lima.

Ragúz, M. (1993d). Socialización de los roles sexuales y las relaciones de

género. Ponencia en el curso del Diploma de Estudios de Género,

Teorías de las Relaciones de Género, Lima: PUCP. Publicación interna.

Ragúz, M. (1992a). Sexualidad humana. Material de apoyo, CENDOC Psicología PUC, Lima (Libro en preparación, 1994).

Ragúz, M. (1992b). Identidad femenina, maternidad y roles sexuales. Ponencia en el Taller del Movimiento M. Ramos para Promotoras de INNPARES, Lima, Mayo 23.

Ragúz, M. (1992c). Amor... "Más te pego, más te quiero"?. Columna Médula Espinal. Revista Amanda, 1, Lima.

Ragúz, M. (1991a). Masculinity and femininity: An empirical definition. Tesis doctoral en Psicología, Universidad Católica de Nimega, Holanda.

Ragúz, M. (1991b). Existe el "eterno femenino"? Ponencia en Mesa Redonda del Diploma de Estudios de Género, CCSS PUC, Lima Oct. 2.

Ragúz, M. (1991c). Adquisición de la masculinidad y femineidad a lo largo de la vida. Ponencia en el V Congreso Peruano de Psicología, Lima, Set. 3 .

Ragúz, M. (1991d). Roles sexuales y anticoncepción. Ponencia en Congreso Peruano de Planificación Familiar, Lima, Set.25.

Ragúz, M. y Bendezú, A. (1992). Adolescentes de condición baja y embarazo temprano: Efectos de variables psicosociales y demográficas sobre la conducta de riesgo reproductivo en escolares diurnos, nocturnos y no escolares. 
Proyecto de investigación aprobado por el Programa especial de Investigación, Desarrollo y Entrenamiento en Investigación en Reproducción Humana, OMS, 1994-96.

Ragúz, M. y Pinzás, J. (1994). Life in Perú. Capítulo XVII del International Handbook of Sex Roles de E. Loeb (ed), Greenwood Press.

Ramos, M.C. (1987). Estereotipos de rol sexual y estándar de salud mental en un grupo de psicoterapeutas y estudiantes universitarios. Tesis de Bachillerato en Psicología, Lima: PUCP.

Rodriguez, G. (1993). Experiencias de género en los programas de planificación familiar en América Latina. México: Mexfam, Pathfinder Int. y The Population Council.

Williams, J. y Best, D. (1982). Measuring sex stereotypes. California: Sage. 